\title{
ÜBER DIE RÜCKBILDUNG DER KIEMENBOGEN BEI DEN SELACHII
}

\author{
VON
}

\author{
J. VERSLUYS, \\ HILVERSUM.
}

(Mit 7 Figuren im Text)

Das Vorkommen von sieben Kiemenbogen bei Heptanchus, sechs bei Hexanchus, Chlamydoselachus und Pliotrema (REGAN, 1908), und von nur fünf bei den übrigen Selachii weist auf Rückbildung der Zahl der Kiemenbogen innerhalb der Selachii hin. Dadurch entsteht die Frage wie diese Verringerung der Zahl der Kiemenbogen vor sich ging. Es wird allgemein angenommen dass diese von hinten her erfolgte ${ }^{1}$ ), dass also die 5 Kiemenbogen der pentanchen Selachii dem $1^{\text {sten }}$ bis $5^{\text {ten }}$ Bogen der Notidani und von Pliotrema entsprechen. Für diese Auffassung spricht das Auftreten von Rudimente weiterer, hinter dem letzten gut entwickelten Kiemenbogen liegender Kiementaschen und Skeletbogen, und weiter auch die Tatsache, dass die Rückbildung von hinten her noch bei höheren Formen wie die Selachii verfolgt werden kann ${ }^{2}$ ). So finden wir bekanntlich Reste des Skeletes eines $6^{\text {ten }}$ Kiemenbogens bei den pentanchen Haien (bei Heterodontus nach HawkES, 1905, und Daniel, 1915, p. 463; vielleicht einen Rest auch bei vielen anderen Formen, vergl. Gibian, 1913, p. 90, und über Stegostoma LuThER, 1909, p. 15), eines $7^{\text {ten }}$ Bogens bei Hexanchus (K. FüRBRINGER, 1903, p. 415) und bei Chlamydoselachus (K. Fürbringer, 1903, p. 413; HAwKes, 1907, p. 478; Goodey, 1910, p. 548). Und wenn auch die Angaben über weitere Kiementaschen nicht immer bestätigt wurden (vergl. BEARD, 1886, p. 108-109; C. K. Hoffmann, 1897 , p. 387 ; van Bemmelen, 1885, p. 186; Greil, 1905, p. 496), so ist doch im Suprapericardial-Körperchen, wie der Entdecker vAN BEMMELEN $(1885 ; 1888$; 1889) richtig hervorgehoben hat, ein Organ gegeben, welches aus einem rudimentär gewordenen Kiemenspalt, der hinter dem letzten gut ausgebildetem lag, hervorgeht (ultimobranchial Körper, GREIL, 1905; postbranchiale Körper, MAURER, 1887; vergl. CAMP, 1917).

So weisen einige Tatsachen ganz bestimmt darauf hin, dass bei den Stammformen der Elasmobranchii die Zahl der typischen Kiemenspalten mehr wie $5^{3}$ ) war, sogar mehr wie 7 oder 8 , da Heptanchus embryonal neben den 7 normalen Kiemenspalten noch einen rudimentären und dann noch ein Suprapericardial-Körperchen aufweist; also, ohne das Spritzloch, 9 Kiemenspalten (BraUs, 1906). Eine grössere Zahl Kiemenspalten weisen bekanntlich unter den Chordata noch Amphioxus (wo allerdings mit einer sekundären Vermehrung gerechnet werden muss) und die Myxinoiden (Bdellostoma, ${ }^{4}$ ) auf.

Und das Nächstliegende ist es dann wohl auch innerhalb der Selachii eine Verringerung der

1) Gegennauder, 1872, p. 277 und 1898, p. 418, 419, 421; MAurer, 1887, p. 374; M. FürBRINGeR, 1897, p. 654 und 691 ; BüTSCHLI, 1910, p. 242; WIEDERSHEIM, 1906, p. 89 und 447, Fussnoten.

2) Bei den Holocephalen ist der letzte Kiemenspalt der $4^{\text {te }}$; der $5^{\text {te }}$ ist verschlossen, also ganz rudimentär geworden; bei Amphibia und Reptilia erfolgt Rückbildung von hinten her bis auf 4 Bogen.

3) Wir zählen das Spritzloch nicht mit, weil es nicht immer vorhanden ist und eine Sonderstellung einnimmt.

4) Hier ist embryonal Rückbildung nachgewiesen, die aber nicht von hinten, sondern von vorne her erfolgt (PRICE, 1906 A und B; M. Fürbringer, 1897, p. 620. 
Zahl der Kiemenbogen und Spalten an zu nehmen, wodurch von hinten her allmählig die Zahl der Kiemenbogen von 7 bei Heptanchus auf 6 und schliesslich auf 5 herabgesetzt wurde.

Die gleiche allmählige Rückbildung von hinten her muss dann auch für die mit dem Kiemenskelet verbundene Muskulatur und für die Nerven und Blutgefässe dieses Gebietes angenommen werden.

Suchen wir nun bei pentanchen oder hexanchen Haifischen nach den Etappen dieser Rückbildung, so finden wir überraschend wenig Anweisungen davon. Man bekommt den Eindruck, dass der

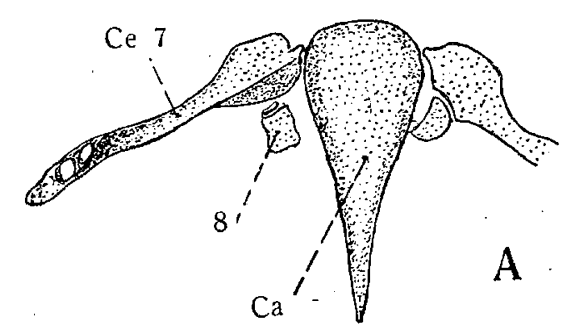
jedesmal in Rückbildung befindliche letzte Bogen und Kiemenspalt sehr schnell bis auf einen sehr kleinen Skeletrest verschwinden.

Vergleichen wir z. B. Hexanchus und Heptanchus. Da diese Gattungen einander sehr nahe stehen, würde man erwarten dass vom $7^{\text {ten }}$ Bogen des Heptanchus bei Hexanchus noch erhebliche Skeletreste übrig seien. Dies ist aber nicht der Fall; der $7^{\text {te }}$ Bogen von Hexanchus ist sehr rudimentär, keinesfalls grösser als der rudimentäre $8^{\text {te }}$ Bogen von Heptanchus (Fig. 1 A und B). Auch

B Chlamydoselachus zeigt nur sehr geringe Reste des $7^{\text {ten }}$ Kiemenbogens

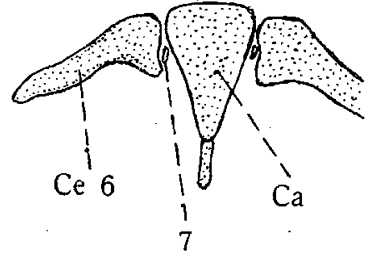

Fig. 1. A. Rudimentärer, $8^{\text {ter }}$ Kiemenbogen von Heptanchus. Nach K. FüRBRINGER, 1903, tab. 17, Fig. 22. B. Rudimentärer, $7^{\text {ter }}$ Kiemenbogen von Hexanchus. Nach K. FürBRINGER, 1903, tab. 17 Fig. 19. $\mathrm{Ca}$ Cardiobranchiale; $\mathrm{Ce} 7$ und Ce 6 letztes Ceratobranchiale; 8 bezw. 7 rudimentärer letzter Kiemenbogen. seiner heptanchen Vorfahren (Fig. 2). Vergleichen wir nur die pentanchen Haie, so finden wir hier nirgends noch sehr erhebliche Reste des $6^{\text {ten }}$ Kiemenbogens. Meist fehlt derselbe gänzlich oder ist doch nur als ein kleiner Fortsatz des $5^{\text {ten }}$ Ceratobranchiale erhalten (GiBian, 1913, p. 90; LuTHER, 1909, p. 15). Sicher vorhanden und etwas weniger rudimentär ist der $6^{\text {te }}$ Kiemenbogen nur bei den Heterodontidae (HAwkEs, 1905; DANIEL, 1915), wo er noch zwei Stücke aufweist (Fig. 3).

Am meisten überraschend ist in dieser Hinsicht wohl Pliotrema, die Pristiophoride mit 6 gut entwickelten Kiemenbogen und Spalten (das Spritzloch nicht mitgerechnet). Diese Form steht Pristiophorus sehr nahe; wie aus REGAN's Beschreibung (1908) hervorgeht, ist der Unterschied in der Zahl der Kiemenbogen und Spalten eigentlich der einzige wesentliche Unterschied der beiden Gattungen, der bei Betrachtung von Aussen hervortritt. Man würde nun erwar-

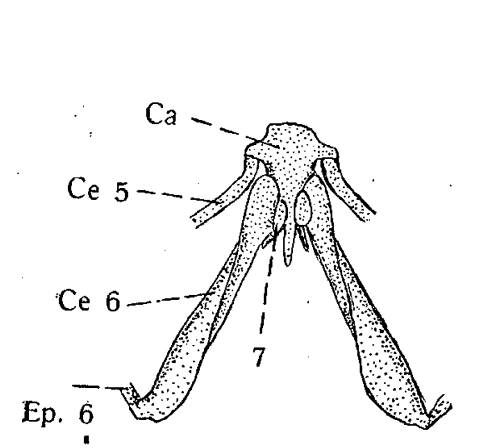

Fig. 2. Rudimentärer, 7ter Kiemenbogen von Chlamydoselachus. Nach Goodey, 1910, tab. 43, rig. 6. Ca Cardiobranchiale; Ce 5 und $C e \quad 6$ fünftes und sechstes Ceratobranchiale; $E p 6$ sechstes Epibranchiale; 7 rudimentärer letzter Kiemenbogen.

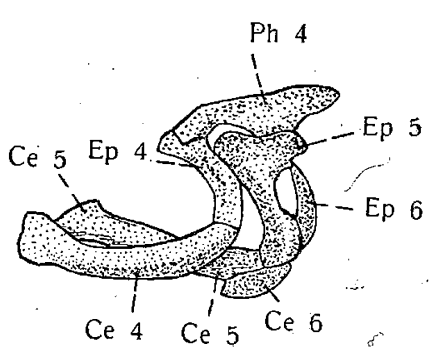

Fig. 3. Der $4^{\text {te }}, 5^{\text {te }}$ und der rudimentäre 6 te Kiemenbogen von Heterodontus francisci, inke Sei-

ie, von vorne unu von der Seite gesehen. Nach DANIEL, 1915, tab. 4, Fig. 14. $\times \frac{1}{2}$. Ce 4,5,6, viertes, fünftes und rudimentäres sechstes Ceratobranchiale; $E p$ 4, 5, 6 , viertes, fünftes und rudimentäres sechstes Epibranchiale; $P h 4$ viertes Pharyngobranchiale. ten, dass Pristiophorus noch einen erheblichen Rest des $6^{\text {ten }}$ Kiemenbogens von Pliotrema aufweist. Aber es zeigt sich, dass überhaupt kein Rest dieses Bogens mehr vorhanden ist (L. HofFMANN, 1914, p. 194); er ist trotz der nahen Verwandtschaft beider Gattungen, bei Pristiophorus gänz.lich ver$\mathrm{schw}$ unden. Ein solches Verhalten ist, wenn man eine allmählige, von hinten her stattfindende Rückbildung annimmt, doch wohl sehr unerwartet.

Auch die Lage des Suprapericardial-Körperchens (vergl. Fig. 4, $\mathrm{SK}$ ) gibt uns Anweisungen zur Beurteilung der Art der Rückbildung, da es sich aus dem Epithel eines rudimentären Kiemenspaltes bildet, der, wie oben hervorgehoben, hinter dem letzten gut ausgebildeten Kiemenspalt liegt. Bei Heptanchus wurde es beim Embryo gefunden (Braus, 1906) als Derivat eines $9^{\text {ten }}$ Kiemenspaltes (das Spritzloch nicht mitgezählt), da zwischen dem Suprapericardial-Körperchen und dem letzten $\left(7^{\text {ten }}\right)$ normalen Kiemenspalt noch ein rüdimentärer Spalt liegt. Bei den pentanchen Haien sollten also zwischen dem letzten gut entwickelten, $5^{\text {ten }}$ Kiemenspalt und 
dem Suprapericardial-Körperchen neben dem rudimentären $8^{\text {ten }}$ Kiemenspalt von Heptanchus noch der $6^{\text {te }}$ und $7^{\text {te }}$ Spalt, wenn auch als Rudimente, vorhanden sein. Dies stimmt aber nicht; das Suprapericardial-Körperchen entsteht hier aus dem Epithel der $6^{\text {ten }}$ Kiementasche (das Spritzloch nicht mitgezählt)! Die zwischen dem letzten, $5^{\text {ten }}$ Kiemenspalt und dem Suprapericardial-Körperchen zu erwartenden Rudimente der ehemaligen $6^{\text {ten }}$ und $7^{\text {ten }}$ Kiementaschen fehlen vollständig, und man muss annehmen, dass das Suprapericardial-Körperchen sich bei den pentanchen Haien aus einer anderen Kiementasche entwickelt als bei Heptanchus, wenn man eine von hinten nach vorne fortschreitende Rückbildung der hinteren Kiementaschen als feststehend betrachtet. Es sei bemerkt, dass bei Pristiophorus das Suprapericardial-Körperchen genau die gleiche Lage, unmittelbar hinter dem Ceratobranchiale 5 aufweist, wie bei anderen pentanchen Formen (L. HoffmanN, 1914, p. 205).

Es liegt bei den Haifischen, wenn wir vom sehr stark rüdimentären $8^{\text {ten }}$ Spalt bei Heptanchus absehen, das Suprapericardial-Körperchen immer direkt hinter dem letzten gut entwickelten Kiemenspalt, ob dieser nun der $5^{\text {te }}$ oder $6^{\text {te }}$ oder $7^{\text {te }}$ sei $\left.{ }^{1}\right)$. Das heisst, statt dass rudimentäre Kiementaschen uns Beweise einer allmähligen Rückbildung Iiefern, konstatieren wir absolutes Verschwinden der betreffenden, vor dem Suprapericardial-Körperchen von Heptanchus liegenden Spalten und konstante Lage des Suprapericardial-Körperchens zum letzten gut entwickelten Kiemenbogen, unabhängig von der Zahl der gut ausgebildeten Kiementaschen und Bogen.

Wir finden also, dass grössere Reste des $7^{\text {ten }}$ b. z. w. $6^{\text {ten }}$ Kiemenbogens und Kiemenspaltes fehlen, die man bei hexanchen und pentanchen Haien doch häufiger finden müsste, falls eine allmählige Rückbildung des Kiemenkorbes von hinten her statt gefunden hätte. Dies tut Zweifel an der Richtigkeit dieser Auffassung einer innerhalb der Selachii von hinten fortschreitenden Rückbildung aufkommen.

Und noch eine Ueberlegung spricht gegen diese Auffassung. Sie setzt voraus, dass der letzte Kiemenbogen bei pentanchen, hexanchen und heptanchen Haien ein anderer ist - und dann müsste dieser Bogen auch einen verschiedenen Bau bei diesen drei Stufen der Rückbildung des Kiemenskeletes aufweisen. Es könnte der letzte Skeletbogen bei den pentanchen Haien doch erst allmählig die Anpassungen erwerben, die der letzte Bogen bei den hexanchen Formen im Zusammenhang mit der Anlehnung am Schultergürtel zeigt. Aber auch in diesem Falle ist
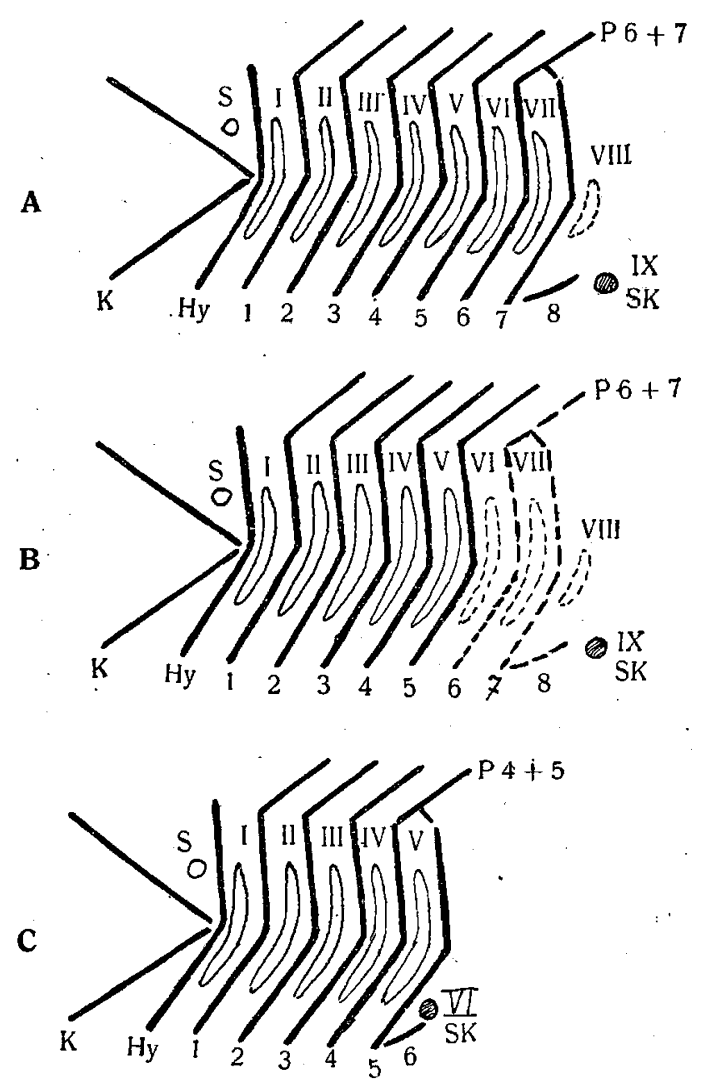

Fig. 4. Schemata zur Demonstration der Lage des Suprapericardial-Körperchens: A bei Heptanchus; $\mathrm{B}$ bei einem pentanchen Hai wenn die rückgebildeten Kiemenbogen und Taschen noch angelegt wurden; C wirkliche Verhältnisse bei pentanchen Haien.

Rudimentär gewordene Spalten und Skeletbogen sind in Schema B mit durchbrochenen Linien angegeben; die Kiemenbogen sind numeriert 1 bis 8 , die Kiemenspalten I bis IX. $H y$ Hyoidbogen; $K$ Kieferbogen; $\mathrm{P} 6+7$ bzw. $\mathrm{P}$ $4+5$ T-förmiges, letztes Pharyngobranchiale; $S$ Spritzloch; SK Suprapericardial-Körperchen (Derivat des $9^{\text {ten }}$ bezw. $6^{\text {ten }}$ Kiemenspaltes).

keine einzige Andeutung einer allmähligen Umbildung und Anpassung des $5^{\text {ten }}$ Bogens an den neuen Verhältnissen zu finden; der letzte Bogen der pentanchen Formen zeigt genau die gleichen Anpassungen, wie der letzte Bogen der hexanchen Formen. Sehr auffällig ist dies bei Prtstiophorus, wo der letzte Bogen durchaus bis in allerlei Einzelheiten dem letzten von Pliotrema gleicht und genau die gleichen Spezialisationen aufweist. Und dasselbe finden wir beim Vergleich des letzten $\left(6^{\text {ten }}\right.$ b. z.w. $\left.7^{\text {ten }}\right)$ Kiemenbogens von Hexanchus und Heptanchus (Fig. 5, A und B). Der letzte Kiemenbogen weist überall annäherend die gleiche Spezialisation und gleich weit gehende Anpassung auf, unabhängig davon ob es der $5^{\text {te }}, 6^{\text {te }}$ oder $7^{\text {te }}$ Kiemenbogen ist. Der $5^{\text {te }}$ Bogen der pentanchen Haien gleicht nicht dem $5^{\text {ten }}$ 
der hexanchen oder heptanchen, sondern dem $6^{\text {ten }}$ b. z.w. $7^{\text {ten }}$ ! Allgemein $\left.{ }^{1}\right)$ schliessen sich das Epibranchiale und Pharyngobranchiale des letzten Bogens dem Pharyngobranchiale des vorletzten Bogens

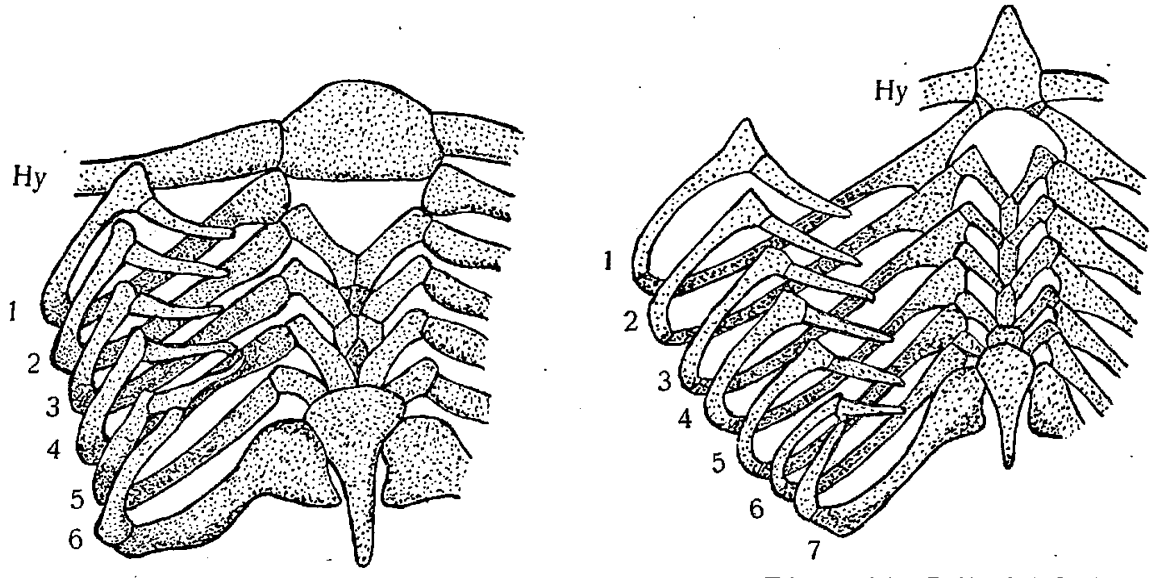

Fig. 5. A Kiemenskelet von Hexanchus, von oben. Die rechte Seite ist fortgelassen. Nach GegenBaur, 1872, tab. 18, Fig. 2.

B Kiemenskelet von Heptanchus, von oben. Die rechte Seite ist fortgelassen. Nach GegenBauR, 1872, tạb. 18, Fig. 1.

Hy Hyoidbogen. Die Kiemenbogen sind numeriert 1 bis 7 . Der rudimentäre letzte Kiemenbogen ist nicht angegeben. an, sodass ein sehr typisches etwa T-förmiges Knorpelstück entsteht (VAN DEINSE, 1916, p. 438 f.; L. HofFMAnN, 1914 p. 190,191 ; auch bei Heterodontus, vergl. Fig. 3, Ph. 4). Die Figuren 5 und 6 geben ein klares Bild dieser Verhältnisse; man vergleiche auch Fig. $4, P 6+7$ und $P 4+5$. Bemerkenswert ist weiter das Verhalten des Musculus trapezius. Bei Heptanchus geht ein vorderer, kleiner Teil desselben nicht zum Schultergürtel, sondern zum oberem Ende des letzten, also $7^{\text {ten }}$, Kiemenbogens

(Fig. 7, Tr. pr.); Insertionen des Trapezius am $6^{\text {ten }}$ oder $5^{\text {ten }}$ Kiemenbogen bestehen nicht (VETTER, 1874, p. 412). Man würde nun erwarten, dass diese besondere Muskelportión mit dem Schwund des $7^{\text {ten }}$ Bogens gleichfalls der Rückbildung anheimfallt und verschwindet. Das ist aber nicht der Fall. Genau wie bei Heptanchus ein vorderer Abschnitt zum letzten, $7^{\text {ten }}$, Bogen zieht, geht bei Chlamydo-

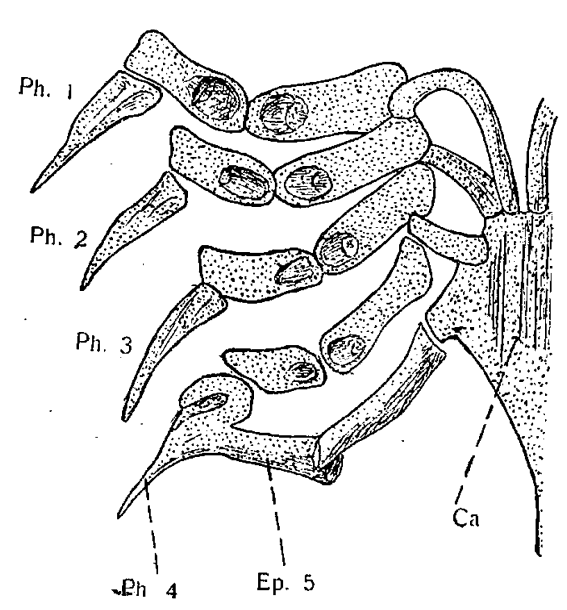

Fig. 6. Kiemenskelet von Pristiophorus japonicus, dorsaie Ȧnsicht mit nach links geklappten Epi- und Pharyngobranchialia. Nach L. HoFFMANN, 1914, tab. 10, Fig. 3

Ph. 1 bis $P h .4$ erstes bis viertes Pharyngobranchiale. Mit $P h .4$ sind das $5^{\text {te }}$ Pharyngobranchiale und Epibranchiale $(E p .5)$ in der üblichen Weise zu einem T-förmigen Stücke verwachsen. $\mathrm{Ca}$ Cardiobranchiale.

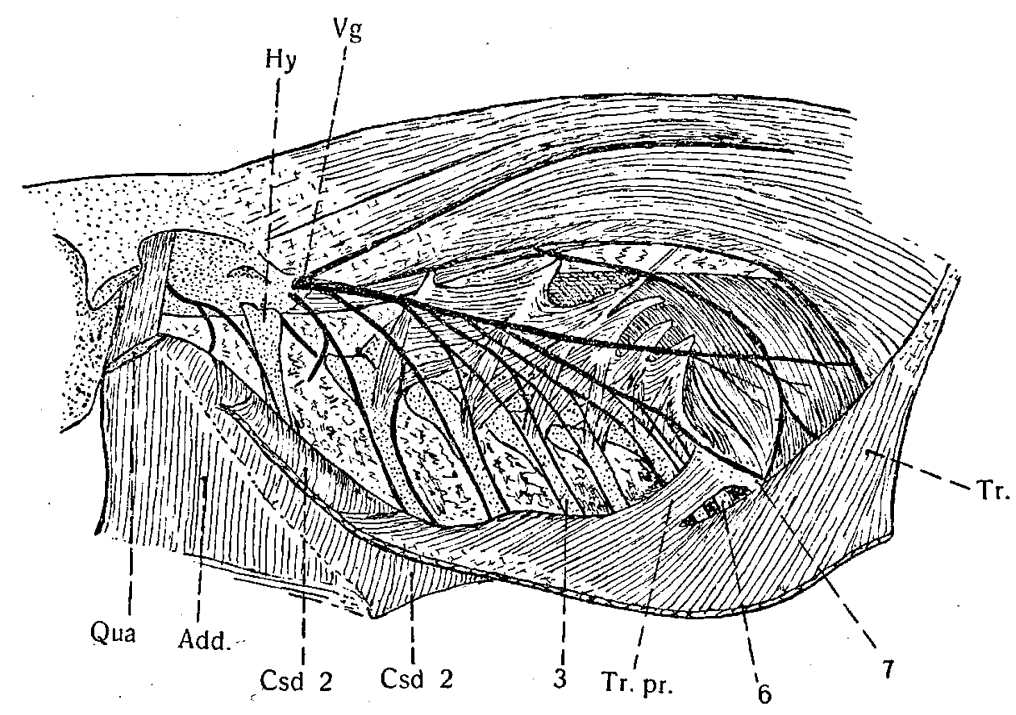

Fig. 7. Der Musculus trapezius bei Heptanchus, linke Seite. Nach VETTER, 1874, tab. 14, Fig. 2. Der $M$. trapezius und andere oberflächliche Muskeln sind dorsal losgelöst und ventralwärts zurückgeschlagen. Knorpel punktiert; Nerven schwarz.

Add M. adductor mandibulae; Csd 2 Portion des dorsalen Abschnittes des M. constrictor superficialis; Hy Hyoidbogen; Qua Quadratteil des Oberkiefers; $T r M$. trapezius; $T r$. $p r$ Insertion des $M$. trapezius am letzten (7ten) Kiemenbogen (M. trapezius profundus); $V g$ Nervus vagus; 3, 6, 7 dritter, sechster und siebenter Kiemenbogen.

selachus (Allis, 1917, p. 344) eine Portion zum sechsten Bogen, bei pentanchen Haien (Scymnus, Vetter, 1874; Acanthias, Vetter; Marion, 1905, p. 20; Mustelus, Tiesing, 1896, p. 113; Stegostoma, Luther, 1909, p. 24) und sogar bei den Holocephalen (Chimaera nach VeTTER, 1878, p. 445, 460) eine Portion, der M. trapezius profundus von VETTER, zum $5^{\text {ten }}$, also jedesmal zum letzten Kie-

1) Vereinzelte Ausnahmen finden wir nur bei pentanchen Haien, z. B. bei Squatina und soweit aus GARMAN's Beschreibung (1885-86) ersichtlich ist, bei Chlamydoselachus. 
menbogen. Eine allmählige Umbildung des Kiemenkorbes von hinten her, mit Rückbildung des $7^{\text {ten }}$ und $6^{\text {ten }}$ Kiemenbogens, setzt eine kompliziertere Umbildung dieses $M$. trapezius profundus voraus (vergl. M. FürBRINGER, 1897, p. 724, namentlich die Fussnote).

GegenbauR hat diese Konstanz des letzten Kiemenbogens sehr richtig beobachtet. Er schreibt darüber (1898, p. 421): „Diese Veränderung des letzten Bogens steht mit seiner Function in engstem "Zusammenhang. Die letzte Kiementasche, welche er hinten stützt, trägt hier keine Kieme mehr, und "manche neue Beziehung zur Nachbarschaft kommt zum Ausdruck. Es ist die Verbindung mit dem „Schultergürtel, welche hier wirksam ward. Der letztere empfängt vom letzten Branchiale eine Stütze. "Solche Verhältnisse treffen den letzten Bogen ohne Rücksicht auf die Zahl der "vorhergehenden '). Heptanchus zeigt sie am 7., Hexanchus und Chlamydoselache am 6., die „übrigen Haie und Chimaera am 5. Bogen." Gegenbaur fährt dann fort: „Wenn wir annehmen dür"fen, dass alle einem gemeinsamen Stamme entsprungen sind, so ist bei Hexanchus der 7. Bogen ver"schwunden und der 6 . hat seine Form angenommen, sowie bei den übrigen der 6 . verloren ging und „der 5. mit dessen functionellen Beziehungen auch die betreffende Umgestaltung empfing. Die Onto"genese hat dafür bis jetzt die Nachweise versagt; sie ist darin wenig sicher, ob. z. B. dem Zustande „des 5. Bogens einmal ein solcher grösserer Ausbildung voranging."

Es ist aber klar, dass dieser nahezu unveränderliche Bau des letzten Kiemenbogens, die Tatsache, dass er immer seiner besonderen Funktion als letzter Bogen gleich vollkommen angepasst ist, mitsamt seiner Muskulatur (Portion des.M. trapezius), sich nicht verträgt mit der Auffassung, dass in dieser Region so weitgehende Umbildungen stattgefunden haben, wie die allmählige Rückbildung und die Ersetzung des $7^{\text {ten }}$ Bogens als letzten durch den $6^{\text {ten }}$, und dieses Bogens durch den $5^{\text {ten }}$ sie bedingen müssten. Und dass hinter dem $5^{\text {ten }}$ oder $6^{\text {ten }}$ Bogen der pentanchen und hexanchen Formen niemals wirklich erhebliche Reste des eben ausser Funktion gesetzten $6^{\text {ten }}$ b. z.w. $7^{\text {ten }}$ Kiemenbogens und Spaltes gefunden werden, steht gleichfalls nicht im Einklang mit der postulierten Rückbildung. Zwar finden wir Reste dieses Skeletbogens und im Suprapericardial-Körperchen auch einen Rest eines reduzierten Kiemenspaltes, aber diese Reste sind schon bei Heptanchus vorhanden und zwar in annäherend gleicher Beziehung zum letzten Kiemenbogen und Kiemenspalt wie bei den pentanchen oder hexanchen Selachii. Der kleine Knorpelrest des $7^{\text {ten }}$ Bogens bei Hexanchus ist in Grösse und Lage so sehr ähnlich dem des $8^{\text {ten }}$ Bogens bei Heptanchus, dass vielmehr an den Rest des gleichen Kiemenbogens, an ein homologes Gebilde gedacht werden muss, ebenso wie das Suprapericardial-Körperchen, trotz seiner verschiedenen Lage in Bezug auf die Zahl der vorangehenden Kiemenspalten, wohl bei allen Selachii ein homologes Organ ist. Nur so ist erklärlich, dass dieser rudimentäre Kiemenbogen bei pentanchen, hexanchen und heptanchen Haien immer ziemlich gleich ausgebildet ist.

Der ganze Organ-komplex am Ende des Kiemenkorbes: der letzte gut ausgebildete Kiemenbogen, der eventuel dahinter vorhandene Rest eines weiteren Bogens ${ }^{2}$ ), der letzte Kiemenspalt, der Schultergürtel, der. Musculus trapezius, das Suprapericardial-Körperchen - zeigt bei allen Haifischen annäherend den gleichen spezialisierten b.z.w. rückgebildeten Bau und demnach muss ein Aufbau dieses Komplexes aus homologen ${ }^{3}$ ) Teilen angenommen werden. Es kann nicht richtig sein, dass innerhalb der Selachii der $7^{\text {te }}$ Kiemenbogen allmählig zurückgebildet und funktionell vom $6^{\text {ten }}$ ersetzt wurde, dieser dann wieder bei den meisten vom $5^{\text {ten }}$ Bogen. Bei so nahestehenden Formen wie Heptanchus und Hexanchus, oder Pliotrema und Pristiophorus müsste man noch deutliche Spuren dieser Umbildung, namentlich eine weniger vollkommene Anpassung des letzten Bogens an seiner neuen Aufgabe bei Hexanchus b. z. w. Pristophorus finden ${ }^{4}$ ).

1) Sperrung von mir.

2) Das die Rudimente dieses weiteren Bogens bei den Heterodontidae grösser sind wie bei den Notidani, kann dadurch erklärt werden, dass jene Formen hierin, wie in anderen Punkten, ursprüngiichere Verhältnisse bewahrt haben, wie diese. Es ist dies kein Beweis gegen die Homologie dieses Bogenrestes mit dem bei Notidani vorhandenen Rudiment eines $7^{\text {ten }}$, bzw. $8^{\text {ten }}$ Bogens.

3) Wir nehmen hier den Begriff Homologie im weiteren Sinne; vergl. die Fussnote 3 auf Seite 101.

4) Es ist bedauerlich, dass die systematische Stellung und die Anatomie der Kiemengegend von Pentanchus, der nach SMITH (1912) ein Notidanide mit nur 5 Kiemenspalten ist, noch nicht genauer bekannt sind. Falls Pentanchus ein Notidanide ist (WILDER, 1913, bestreitet dies), würde das Vorkommen von 7, 6 und 5 Kiemenspalten und Bogen innerhalb einer Familie des Selachii sehr zu Gunsten einer leichten Aenderung dieser Zahl sprechen, wie sie mit einer allmähligen Rückbildung und Umbildung von hinten her nicht vereinbarlich wäre. 
Auch funktionell ist eine Ersetzung des $7^{\text {ten }}$ Bogens als Abschluss des Kiemenkorbes durch den $6^{\text {ten }}$, und dann durch den $5^{\text {ten }}$ unverständlich. Der letzte Bogen hat immer die wichtige Aufgabe, den Schultergürtel am Kiemenkorb zu fixieren und umgekehrt; er ist dieser Funktion angepasst und unterscheidet sich entsprechend im Bau von den vorhergehenden Kiemenbogen. Warum soll dann aber dieser Bogen bei heptanchen und hexanchen Selachii der allmähligen Rückbildung anheim fallen um vom vorhergehenden Bogen, der dabei ziemlich genau seine Form annimmt, ersetzt zu werden? Und wie soll ein funktionell brauchbarer Uebergangszustand geschaffen werden bevor an Stelle des der Rückbildung verfallenen letzten Bogens der vorletzte Bogen die notwendigen Anpassungen in seiner Gestalt und seiner Muskulatur erreicht hat? Eine solche Umbildung war, nachdem einmal bei den Vorfahren der Selachii der Anschluss des letzten ( $7^{\text {ten }}$ Kiemenbogens am Schultergürtel mit allen entsprechenden Spezialisationen erreicht worden war, funktionell schwierig und zwecklos, weil sie durch einen weniger angepassten, also weniger geeigneten Uebergangszustand hindurch gehen musste. Die Bedeutung dieser Anpassung geht daraus hervor, dass sie beim letzten Kiemenbogen immer annäherend die gleiche ist, ob dieser Bogen nun der $5^{\text {te }}, 6^{\text {te }}$ oder $7^{\text {te }}$ sei.

Die Verringerung der Zahl der Kiemenbogen und Spalten muss demnach innerhalb der Selachii in anderer Weise vor sich gegangen sein, als durch allmählige Rückbildung von hinten her.

An eine von vorne her einsetzenden Rückbildung ist aber auch nicht zu denken; hier bietet uns der Komplex Kieferbogen-Hyoidbogen genau so einen festen Punkt, wie der letzte Kiemenbogen am hinteren Ende des Visceralskeletes. Und nirgends finden wir bei den Selachii Anweisungen einer allmähligen Rückbildung eines Bogens innerhalb der Reihe der Kiemenbogen ${ }^{1}$ ), zwischen den spezialisierten vorderen und hinteren Visceralbogen. Die Verringerung der Zahl der Kiemenbogen muss demnach ohne Uebergangszustände statt gefunden haben; plötzlich muss der Zustand mit 6 Kiemenbogen aus dem heptanchen, und der pentanche Zustand aus dem hexanchen hervorgegangen sein, sowohl bei den pentanchen Nachkommen der Notidani wi e bei Pristiophorus.

Wir wissen, dass ein metameres Organ in verschiedener Zahl ausgebildet sein kann. Namentlich die Regionen der Wirbelsäule bieten davon Beispiele. Ein für den Vergleich mit den Kiemenbogen besonders interessanter Fall ist die von Bolk (1900) beschriebene menschliche Halswirbelsäule mit 6 statt 7 Wirbeln, während diese Wirbelsäule in Uebrigen durchaus normal ist; der $7^{\text {te }}$ Wirbel ist hier zweifellos der normale erste Brustwirbel, nicht ein abnormaler Halswirbel. Einen ähnlichen Fall erwähnt BATESON (1894, p. 116, unter $\mathrm{N}^{\circ} 33$ ) von einem Chimpansen, wo 12 statt 13 Brustwirbel vorhanden sind, neben einer normalen Hals-, Lumbal- und Sacralregion. Auch bei Choloepus finden wir bekanntlich den $7^{\text {ten }}$ Wirbel als ersten Brustwirbel ausgebildet (SOLGER, 1876; WELCKER, 1878; BATESON, 1894, p. 118 f.). Aehnlich sind selbstverständlich Fälle zu verwerten, wo eine Vermehrung der Wirbelzahl einer bestimmten Region statt gefunden hat, wie an der Halswirbelsäule von Bradypus (SolGER; WELCKER; BATESON) oder an der Wirbelsäule von Gavialis gangeticus und Heloderma (BAUR, 1891). Am auffälligsten ist wohl die Wirbelvermehrung bei der Halswirbelsäule-einiger Plesiosaurier, worauf BAUR (1891; 1897) hingewiesen hat.

Bekanntlich ist man nicht einig darüber, wie diese Umbildungen bzw. individuelle Variationen der Wirbelsäule erklärt werden müssen. Es liegen hauptsächlich drei Theorien vor, die alle drei auch für die Umbildung der Zahl der Kiemenbogen Beachtung verdienen ${ }^{2}$ ).

Die erste ist die Theorie RosenberG's, wovon für uns das Wesentlichste ist, dass sie die seriale Homologie der Wirbel annimmt in dem Sinne, dass der 5te Wirbel bei allen Wirbelsäulen homolog ist, ebenso der 10te, 20ste, u. s. w. d.h. es besteht vollkommene Homologie der Wirbel verschiedener Wirbelsäulen, welche einander numerisch entsprechen. Die Form dagegen kann verschieden sein, indem der gleiche Wirbel einmal Sacralwirbel, einmal Lendenwirbel sein kann, u. s. w. (RosenberG, 1899, 1920; M. Fürbringer, 1879).

Eine zweite Auffassung nimmt an, dass Aenderungen in der Zahl der Wirbel eine Folge von Intercalation bzw. Excalation bestimmter Wirbel seien. Der 10te Wirbel einer Wirbelsäule entspricht nicht mehr immer dem $10^{\text {ten }}$ jeder

1) Vergl. Gaupp, 1905, p. 835: "A'usfall eines Bogens aus der Mitte der Reihe heraus ist mit Sicherheit bisher nicht festgestellt".

2) Vergl. auch WinsLow (1904), KingSLEY (1907) und BöHM (1907). 
anderen Wirbelsäule, sondern mitunter dem 9ten oder 11 ten oder noch einem anderen. Es kann die Zahl der Wirbel einer Region (z. B. der Halswirbelsäule im oben erwähnten von BoLK beschriebenen Falle) variiren; indem schon embryonal ein Segment fortfällt bzw. eingeschaltet wird, ohne dass die übrigen Regionen der Wirbelsäule davon berührt werden. Diese Theorie stammt von voN IHERING (1878) und wurde von AlBRECHT (1883; mit einer Aenderung) und dann von BAUR $(1891,1897)$ vertreten.

Eine dritte Theorie nimmt an, dass eine bestimmte Region der Wirbelsäule in einer wechselnden Zahl von Wirbeln auftreten kann, sodass 6 Wirbel einer Wirbelsäule 7 Wirbel einer anderen entsprechen (man denke wieder an die Wirbelsäule mit 6 Halswirbeln von BOLK). Diese Auffassung wurde zuerst von WeLCKER (1878) vertreten, der dabei an einer Nonius-Einteilung erinnert. WELCKER bringt das Wesen seiner Auffassung klar zum Ausdruck indem er sagt, bei einer Wirbelsäule mit 33 Wirbeln und einer mit 34 Wirbeln würde nicht ein intercalierter oder ein UebergangsWirbel vorhanden sein, sondern in den typischen Fällen würde kein einziger Wirbel der einen Wirbelsäule irgend einem der zweiten ganz entsprechen. BATESON (1894), RIDEWOORD (1897) und WAITE (1897) haben sich zu Gunsten dieser Auffassung ausgesprochen; man vergleiche auch DWIGHT (1901) und FISCHEL (1906), die eine ähnliche Auffassung vertreten.

Es ist klar, dass theoretisch alle drei diese Umbildungsweisen neben einander gedacht werden können und sie sich keinesfalls gegenseitig ausschliessen. Und es muss also geprüft werden, welche der drei Theorien bei der Aenderung der Zahl der Kiemenbogen innerhalb der Selachii zutreffend sein könnte. Es fragt sich zunächst, ob man beim Kiemenkorb irgend welche Anhaltspunkte hat um zu entscheiden ob etwa eine Excalation im Sinne IHERING's und BAUR's, oder eine Variation nach dem Prinzip der Nonius-Einteilung im Sinne WelckeR's ') vorliegt. Vergleichen wir unter diesem Gesichtspunkte die Kiemenbogen von Pristiophorus und Pliotrema ${ }^{2}$ ), so finden wir bei beiden die gleiche Spezialisation des ersten Kiemenbogens (ein Hypobranchiale fehlt), sowie des $4^{\text {ten }}$ und $5^{\text {ten }}$ bei Pristiophorus, bzw. des $5^{\text {ten }}$ und $6^{\text {ten }}$ bei Pliotrema (gleiche Spezialisation und Verwachsung der Pharyngobranchialia und des letzten Epibranchiale; besondere Form des letzten Ceratobranchiale; Fehlen des letzten Hypobranchiale). Diese Bogen entsprechen einander so vollkommen in ihrer Ausbildung, dass man sie als homolog betrachten muss. Die Kiemenbogen 2 und 3 von Pristiophorus und 2, 3 und 4 von Pliotrema zeigen keine erheblichen Spezialisierungen oder Differenzen in den Form, wodurch sie individuell gekennzeichnet wären und hier könnte man sowohl an die Excalation eines bestimmten Bogens, wie an einer verschiedenen embryonalen Gliederung, einer Nonius-Einteilung in 2 statt 3 Segmente, denken.

Und das Gleiche gilt auch für Heptanchus, Hexanchus und Chlamydoselachus im Vergleich mit pentanchen Haien. Bei den Notidani ist die Spezialisation der Kiemenbogen gering; nur der erste und die beiden letzten weisen Besonderheiten auf, wodurch sie individiell gekennzeichnet sind (vergl. Gegenbaur, 1872; Garman, 1885/86; K. FürbRINGER, 1903), aber die mittleren Bogen 2 bis 5, bezw. 2 bis 4 sind einander ähnlich und man kann nicht erkennen, dass bei den pentanchen Haien ein bestimmter Bogen (oder zwei bestimmte Bogen von Heptanchus) fehlt.

Es scheint mir sehr schwer zu entscheiden, ob die Rückbildung hier eine Excalation gewesen ist, oder nach dem WeLCKER'schen Prinzip des Nonius-Einteilung vor sich ging. Es ist gerade die Schwierigkeit, dass bei diesen Variationsmodi keine Uebergangsstadien denkbar sind und sie daher nie direkt beobachtet werden können.

Die Theorie RosenBerG's lässt sich vielleicht auch auf das Kiemenskelet übertragen. Zwar ist, wie wir oben darzulegen versuchten, eine allmählige Verschiebung der Spezialisation der Kiemenbogen unter Rückbildung von hinter her auszuschliessen. Aber man könnte doch eine plötzliche Verschiebung nach vorne vertreten, etwa durch die Annahme, die Gestalt der beiden letzten Bogen von Heptanchus sei, infolge einer Verschiebung in der Auswirkung der Gestaltgebenden Faktoren, plötzlich bei den hexanchen Formen auf dem $5^{\text {ten }}$ und $6^{\text {ten }}$ Bogen und ebenso bei den pentanchen auf dem $4^{\text {ten }}$ und $5^{\text {ten }}$ Bogen übertragen worden und dem sei eine entsprechende Verschiebung aller benachbarten Teile (Schultergürtel; rudimentärer letzter Kiemenbogen; Musculus trapezius profundus; SuprapericardialKörperchen!; Ductus Cuvieri) gefolgt ${ }^{3}$ ). Praktisch wäre eine solche Umbildung von der Excalation

1) Die Anffassung von Welcker wurde von RosEnBeRg (1920, p. 51-52) einer beachtenswerten Kritik unterzogen, die aber nur den speziellen Fall der Wirbelsäule betrifft, nicht den hier erörterter, etwas anders liegenden Fall der Rückbildung der Zahl der Kiemenbogen.

2) Ich konnte 1913 ein Skelet von Pliotrema im "British Museum of Natural History" in Londen untersuchen, und möchte Herrn TATE REGAN nochmals an dieser Stelle für sein grosses Entgegenkommen meinen herzlichen Dank aussprechen.

3) M. FürRBRINGER scheint an eine solche Umbildung gedacht zu haben, wie aus seinen Bemerkungen (1897, 
eines der mittleren Bogen oder von einer nach dem Welcker'schen Prinzip stattfindenden Umbildung aber gar nicht zu unterscheiden!

Ich glaube, dass die vorliegenden Tatsachen am einfachsten durch eine Umbildung nach dem WeLCKeR'schen Prinzip erklärt werden können, glaube aber nicht, dass die IHERING'sche oder RosenBERG'sche Auffassung hier ausgeschlossen werden können. Wir brauchen dies hier auch nicht zu entscheiden, da es uns genügt, dass überhaupt eine theoretische Erklärung der oben befürworteten sprungweisen Umbildung des Kiemenkorbes gegeben und mit Analogiefälle belegt werden kann.

Ebenso wie eine plötzliche Verminderung der Zahl der Kiemenbogen und Spalten in der hier dargelegten Weise stattfinden kann, muss theoretisch die Möglichkeit einer Vermehrung zugegeben werden. Der Gedanke, dass bei den Notidaniden eine sekundäre Vermehrung der Zahl der Kiemenbogen und Spalten stattgefunden habe, wurde schon von REIS (1897, p. 88; man vergl, auch VAN WIJHE, 1889) befürwortet. Man muss vor Allem hieran denken, wenn man die systematische Stellung von Pliotrema beachtet. Diese Form ist im Allgemeinen gewiss nicht primitiv; sie steht Pristiophorus so nahe, dass ihre systematische Stellung nicht anders als die von Pristiophorus selbst beurteilt werden kann. Wäre Pristiophorus ein Spinacide, wie es JAEkEl (1891, p. 42) in seiner wichtigen Arbeit ausgesprochen hat, oder gehörten die Pristiophoriden als Unterfamilie zu den Squalidae (REGAN, 1906, p. 733) oder zu Goodrich' Squaliformes (GoOdRICH, 1909, p. 151-152), so wären Pristiophorus und Pliotrema von pentanchen Haifischen abzuleiten und die Sechszahl der Kiemenspalten und Bogen bei Pliotrema wäre eine sekundäre Erscheinung, die nur eine Folge plötzlicher Vermehrung derselben sein könnte. REGAN (1906, p. 740) schrieb über die Zahl der Kiemenspalten der Notidani: „It is probable that their large number is a primitive feature, but the recent discovery of a Pristiophorus with six gillclefts shows that the importance of this character has been overestimated. In this case, however, it is accompanied by a very generalised arrangement of the branchial skeleton."

Und L. HofFMANN schrieb (1914, p. 157): „Es ist ohne weiteres klar, dass dieses Auftreten einer Form mit 6 Kiemenspalten in einer hoch spezialisierten Gruppe von Selachiern ein Licht wirft auf die Entstehung der 6. Kiemenspalte in der Stammesgeschichte derselben und infolgedessen von grosser theoretischer Tragweite sein wird" ').

Aus den Untersuchungen von L. HoffManN $(1912 ; 1914)$ geht aber hervor, dass Pristiophorus doch den Spinaciden oder anderen pentanchen Haien nicht. so nahe steht, wie es JAEKEL annahm. Nach den Ergebnissen von L. Hoffmann ist es möglich, dass die Pristiophoriden direkt auf alte Haifische mit sechs Kiemenbogen und Spalten zurückgehen. Es könnte also Pliotrema in diesem Punkte primitiv geblieben sein, trotz der sonstigen, sehr weit in die Richtung der Rhinoraji gehenden Spezialisierung der Pristiophoriden.

Bei den Notidani haben wir es gewiss mit noch weniger spezalisierten Formen zu tun, wie die Pristiophoriden; besonders ihr Kiemenskelet erscheint durch die Gleichförmigkeit der Skeletbogen und den primitiven Bau der Copularia von einem ursprünglichen Charakter. Die Muskulatur macht hier auch einen primitiven Eindruck. Ich kann denn auch keine Gründe finden, die 6- b. z. w. 7-zahl der Kiemenbogen der Notidani für sekundär zu halten, wenn dies auch theoretisch denkbar wäre.

Nachdenklich stimmt hier allerdings die Tatsache, dass geologisch so alte und primitive Selachier, wie die Ichthyotomi und die Acanthodi nur 5 Kiemenbogen hatten, von den Cladoselachi mehr wie 5 Bogen nicht sicher nachgewiesen wurden, und dass die Holocephali auch nur 5 Kiemenbogen besitzen. Ist die 7-zahl der Kiemenbogen von Heptanchus ein primitives Merkmal, so müssen die Stammformen der Ichthyotomi, Acanthodi, Cladoselachi und Holocephali 7 Bogen aufgewiesen haben und dann ist es befremdend, dass überall eine Rückbildung bis auf 5 Kiemenbogen erreicht wurde; es ist hier von grosser Bedeutung ob die Cladoselachi auch nur 5 Kiemenbogen hatten.

p. 724, Fussnote eins) über die Verlagerung der Insertion des Musc. trapezius am letzten Kiemenbogen hervorgeht. Sein Begriff der imitatorischen Homodynamie wäre hier anwendbar und es würde keine vollständige Homologie aller Teile mehr vorliegen. BATESON (1894, p. 133) spricht für solche Fälle von Homoeosis.

1) L. HoffMANN wollte diese Frage in einer besonderen Arbeit behandeln und ich hatte ihm meine 1913 im British Museum Natural History am Skelet von Pliotrema gemachten Beobachtungen dazu zur. Verfügung gestellt. Leider ist dieser vielversprechende junge Forscher 1914 im Kriege gefallen. 
Man darf vermuten, dass die 6- bzw. 7-zahl der Kiemenspalten bei den Notidani und Pliotrema für diese Tiere von funktionellem Werte ist. Die grössere Zahl der Kiemenspalten und Kiemen könnte eine Vergrösserung der Atemfläche bezwecken; mit einem gesteigertem Bedürfniss nach Atemwasser könnten auch die sehr weiten Kiemenspalten der Notidaniden im Zusammenhang stehen. Die Notidaniden leben soweit bekannt in tieferen Meeresschichten, und der Sauerstoffgehalt pflegt in grösserer Tiefe abzunehmen. Es fehlen uns aber leider genauere Kenntnisse, die es uns ermöglichen würden, die Frage nach der funktionellen Bedeutung der Zahl der Kiemenspalten zu lösen und dadurch fehlt uns ein wichtiger Wegweiser zur Prüfung der Frage ob die höhere Zahl der Kiemenspalten bei den Elasmobranchiern ein alt ererbter, erhalten gebliebener Zustand oder eine neu-erworbene Anpassung ist.

So wie unsere Kenntnisse sind, liegt $\mathrm{m}$. Erachtens kein zwingender Grund vor, die alte Auffassung aufzugeben, dass die höhere Zahl der Kiemenspalten ein ursprünglicher Zustand darstellt. Wohl aber tut die hier dargelegte Theorie von der sprungweisen Umbildung der Zahl der Kiemenbogen innerhalb der Selachii diese alte Auffassung nicht mehr als so fest begründet erscheinen, wie es bisher erscheinen musste, als eine allmählige aber stetig fortschreitende Rückbildung von hinten her die allein gültige Erklärung der verschiedenen Zahl der Kiemenbogen der Selachii schien.

Fassen wir obige Betrachtungen und das Ergebnis zusammen, so kommen wir zu nachfolgender Darlegung.

Wenn auch innerhalb der Fische, auch bei den Stammformen der Selachii, eine allmählige Rückbildung des Kiemenkorbes von hinten her gewiss anzunehmen ist, so fallen doch die Aenderungen in der Zahl der Kiemenspalten und Bogen innerhalb der Selachii nicht unter diesem Gesichtspunkte. Die Umbildung bei den Selachii von 7 auf 6 und 5 Kiemenbogen kann nicht allmählig und von hinten her vor sich gegangen sein, sondern muss sprungweise, ohne Uebergangszustände stattgefunden haben, wobei der Komplex der beiden letzten Kiemenbogen samt Schultergürtel und Muskulatur unverändert blieb, und die Umbildungen in der mittleren Region des Kiemenkorbes stattfanden, es sei denn dass einer der mittleren, nicht besonders differenzierten Bogen verschwand (Excalation), oder dass statt 4 Bogen nur 3 und schliesslich zwei mittlere Bogen ausgebildet wurden. Wie diese Umbildung theoretisch gedacht werden kann, muss dahingestellt bleiben. Alle drei Theorien die zur Erklärung der analogen Umbildungen der Wirbelsäule aufgestellt wurden; kommen in Betracht: die Theorie von ROSENBERG, von Welcker und von von IHERING, wobei die Auffassung WelckeR's im speziellen Falle des Kiemenkorbes vielleicht die einfachste Erklärung gibt.

Die hier vorgebrachte Auffassung hat gewiss etwas Ueberraschendes. Zu ihr wird man geführt durch die Ueberlegung, dass die nahe Verwandtschaft von Heptanchus und Hexanchus, und namentlich von Pliotrema und Pristiophorus eine viel schnellere Umbildung der Zahl der Kiemenbogen bedingt, als die Auffassung einer allmähligen Rückbildung von hinten her gestattet; - weiter spricht für sie die auffallende Gleichförmigkeit des letzten Kiemenbogens bei allen Selachii, dessen Spezialisation und besondere Aufgabe, den Anschluss am Schultergürtel darzustellen und diesen zu stützen, sich einer Rückbildung widersetzen müssen. Und schliesslich sind auch die annäherend gleiche, immer sehr weitgehende Rückbildung des rudimentären $8^{\text {ten }}, 7^{\text {ten }}$ b. z. w. $6^{\text {ten }}$ Kiemenbogens der Notidani und Heterodontidae, das Fehlen von Resten des $6^{\text {ten }}$ Bogens bei Pristiophorus und die Lagebeziehung des Suprapericardial-Körperchens nur in dieser. Weise zu verstehen.

Wenn man eine sprungweise Verringerung der Zahl der Kiemenbogen der Selachii annimmt, wie oben dargelegt, muss auch die Möglichkeit einer sprungweisen Vermehrung der Zahl derselben theoretisch zugegeben werden. Es verdient deshalb die Frage, ob die höhere Zahl der Kiemenspalten der Notidani und von Pliotrema ein primitives Merkmal ist oder nicht, grössere Beachtung, wenn auch vor der Hand kein entscheidender Grund vorliegt, diese Frage im verneinenden Sinne zu beantworten. 


\section{LITERATURVERZEICHNIS.}

Albrecht, P. (1883), Note sur une hémivertèbre gauche surnuméraire de Python sebae, Duméril, in: Bull. Mus. Roy. Hist. Nat. de Belgique, Vol. 2.

Allis, E. P. (1917), The homologies of the muscles related to the visceral arches of the gnathostome Fishes, in: Quart. Journ. microsc. Sci. London, Vol. 62.

BATESON, W. (1894), Materials for the study of Variation. London.

BAUR, G. (1891), The intercalation of vertebrae, in: Journ. Morphol., Voi. 4.

- (1897), Remarks on the question of intercalation of vertebrae, in: Zoolog. Bulletin, Vol. 1

BEARD, J. (1886), The system of branchial sense organs and their associated ganglia in Ichthyopsida, in: Quart. Journ. microsc. Sci., Vol. 26 .

BEMMELEN, J. F. VAN (1885), Ueber vermuthliche rudimentäre Kiemenspalten bei Elasmobranchiern, in: Mittheil. aus der zoolog. Station zu Neapel, Vol. 6 .

- (1888), Over de kieuwspleten en hare overblijfselen bij de hagedissen, in: Donders Feestbundel.

- (1889), Ueber die Suprapericardialkörper, in: Anat. Anz., Vol. 4, p. 400-407.

BöHM, M. (1907), Die numerische Variation des menschlichen Rumpfskelets; Stuttgart.

BoLK, L. (1900), Ueber eine Wirbelsäule mit nur sechs Halswirbeln, in: Morphol. Jahrb., Vol. 29.

BRAUS, H. (1906), Ueber den embryonalen Kiemenapparat von Heptanchus, in: Anat. Anz., Vol. 29.

BüTSCHLI, OTto (1910), Vorlesungen über vergleichende Anatomie. 1ste Liefer., Leipzig.

CAMP, W. E. (1917), The development of the suprapericardial (postbranchial, ultimobranchial) body in Squalus acanthias, in: Journ. Morphol., Vol. 28.

Daniel, J. F. (1915), The anatomy of Heterodontus francisci: II. The endoskeleton, in: Journ. Morphol., Vol. 26.

- 1916), The anatomy of Heptanchus maculatus; the endoskeleton, in: Berkeley Univ. California Public. in Zoology, Vol. 16.

DEINSE, A. B. VAN (1916), Ueber Variationen im Visceralskelett von Acanthias vulgaris; über das Visceralskelett von Lamna cornubica und seine Kalkbedeckung, in: Anat. Anz., Vol. 49.

Dwight, TH. (1901), Description of the human spines showing numerical variation in the Warren Museum of the Harvard Medical School, in: Anat. Anz., Vol. 19.

Fischel, A. (1906), Untersuchungen über die Wirbelsäule und den Brustkorb des Menschen, in: Anat. Hefte, Vol. 31 (Heft 95).

FürbRINGER, K. (1903), Beiträge zur Kenntnis des Visceralskelets der Selachier, in: Morphol. Jahrb., Vol. 31.

FürbRinger, M. (1879), Zur Lehre von den Umbildungen der Nervenplexus, in: Morphol. Jahrb., Vol. 5.

- (1897), Ueber die spino-occipitalen Nerven der Selachier und Holocephalen und ihre vergleichende Morphologie, in: Festschrift f. C. Gegenbaur, Vol. 3, Leipzig.

GARMAN, S. (1885-86), Chlamydoselachus anguineus Garm., in: Bull. Mus. comp. Zool. Harvard College, Vol. 12.

GAUPP, E. (1905), Das Hyobranchialskelet der Wirbeltiere, in: Ergebnisse d. Anat. u. Entwickelungsgeschichte, Vol. $14,1904$.

Gegenbaur, C. (1872), Untersuchungen zur vergleich. Anatomie d. Wirbelthiere, Heft 3, Das Kopfskelet der Selachier, ein Beitrag zur Erkenntniss der Genese des Kopfskeletes der. Wirbelthiere. Leipzig,

_- (1898), Vergleichende Anatomie der Wirbelthiere. Vol. 1. Leipzig.

Gibian, A. (1913), Beiträge zur Kenntnis des Hyobranchialskeletes der Haie, in: Morphol. Jahrb., Vol. 45.

GoODEY, T. (1910), A contribution to the skeletal anatomy of the frilled shark, Chlamydoselachus anguineus Gar., in: Proc. zool. Soc. London, 1910.

Goodrich, E. S. (1909), Cyclostomes and Fishes, in: A treatise on zoology, edited by R. Lankester, Vol. 9, I, London.

GREIL, A. (1905), Ueber die Anlage der Lungen, sowie der ultimobranchialen (postbranchialen, supraperikardialen) Körper bei Anuren Amphibien, in: Anat. Hefte, Vol. 29 (Heft 89).

HAWkes, A. O. MERRIT (1905), The presence of a vestigial sixth branchial arch in the Heterodontidae, in: Journ. Anat. Physiol, Vol. 40.

- ( $[907)$, The abdominal viscera and a vestigial seventh branchial arch in Chlamydoselachus, in: Proc. zoolog. Soc. London 1907, 2.

HofrmanN, C. K. (1897), Beiträge zur Entwicklungsgeschichte der Selachii, in: Morphol. Jahrb., vol. 27.

Hoffmann, L. (1912), Zur Kenntnis des Neurocraniums der Pristiden und Pristiophoriden, in: Zool. Jahrb., Vol. 33, Anat.

- (1914), Das Visceralskelet von Pristiophorus, ibid. Vol. 38

IHERING, H. VON (1878), Das peripherische Nervensystem der Wirbelthiere als Grundlage für die Kenntniss der Regionenbildung der Wirbelsäule. Leipzig.

JaEkel, O. (1891), Ueber die Gattung Pristiophorus, in: Arch. f. Naturgesch., Vol. 57. 
KingsBuRY, B. F. (1915), On the so-called ultimobranchial body of the mammalian embryo: man, in: Anat. Anz., Vol. 47. KINGSLEY, J. S. (1907), Meristic Homologies in Vertebrates, in: Amer. Naturalist, Vol. 41.

Luther, A. (1909), Beiträge zur Kenntnis von Muskulatur und Skelett des Kopfes des Haies Stegostoma tigrinum Gm. und der Holocephalen, in: Acta societatis scient. Fennicae, Vol. 37.

MARION, G. E. (1905), Mandibular and pharyngeal muscles of Acanthias and Raia, in: Amer. Naturalist, Vol. 39.

MAURER, F. (1887), Schilddrüse, Thymus und Kiemenreste bei Amphibien, in: Morphol. Jahrb., Vol. 13.

- (1906), Die Entwicklung des Darmșystems, in: Handbuch d. Entwicklungslehre der Wirbeltiere von O. Hertwig, Vol. 2, I, Jena.

PrICE, G. C. (1896 A), Zur Ontogenie eines Myxinoiden (Bdellostoma Stouti), in: S. B. mathem. phys. Cl. d. Kg. Bay. Akad. d. Wiss., Vol. 26.

- (1896 B), Some points in the development of a Myxinoid (Bdellostoma Stouti Lockington), in: Verhandl. Anat. Ges. zu Berlin 1896 (Ergänzungsband Anat. Anzeiger).

REGAN, C. T. (1906), A classification of the Selachian Fishes, in: Proc. zool. Soc. London, 1906, Vol. 2.

- (1908), Description of new or little known Fishes from the coast of Natal, in: Annals Natal Government Museum.

REIS, O. M. (1897), Das Skelett der Pleuracanthiden und ihre systematischen Beziehungen, in: Abhandl. Senckenbergischen Naturforsch. Gesellschaft, Vol. 20 (1903).

RIDEWOOD, W. G. (1897), On the development of the vertebral Column in Pipa and Xenopus, in: Anat. Anz., Vol. 13. Rosenberg, E. (1899), Ueber eine primitive Form der Wirbelsäule des Menschen, in: Morphol. Jahrb., Vol. 27.

- (1920), Die verschiedenen Formen der Wirbelsäule des Menschen und ihre Bedeutung, 1ster Teil. Jena.

SMith, H. M. (1912), Description of a new notidanoid shark from the Philippine islands, representing a new family, in: Proc. U. St. nation Museum, Vol. 41.

Solger, B. (1876), Zur Anatomie der Faulthiere (Bradypodes), in: Morphol. Jahrb., Vol. 1.

TIEsING, R. (1896), Augen-, Kiefer- und Kiemenmusculatur der Haie und Rochen, in: Jenaische Ztschr. Naturwiss., Vol. 30.

VeTTER, B. (1874), Untersuchungen zur vergl. Anatomie der Kiemen- und Kiefermusculatur der Fische, I, in: Jenaische Ztschr. Naturwiss., Vol. 8 (neue Folge, Vol. 1).

- (1878), ibid., II, ibid., Vol. 12 (neue Folge, Vol. 5)

WAITE, F. C. (1897), Variations in the brachial and lumbosacral plexi of Necturus maculosus Raf., in: Bull. Mus. comp. Zool., Vol. 31 .

WelcKer, H. (1878), Zur Lehre von Bau und Entwicklung der Wirbelsäule, in: Żoolog. Anz., Vol. 1.

WELLS, G. A. (1917), The skull of Acanthias vulgaris, in: Journ. Morphol., Vol. 28

Wiedersheim, R. (1906), Vergleichende Anatomie der Wirbeltiere, 6te Auflage, Jena.

WILDER, B. G. (1913), The brain as a guide to the affinities of Vertebrates, in: Journal Washington Acad. of Sci.; Proceedings $507^{\text {th }}$ regular meeting biolog. Soc. of Washington.

WiNsLow, G. M. (1904), Three cases of abnormality in Urodeles, in: Tufts College Studies №. 8 (Scient. Ser.).

Wijhe, J. W. VAN (1889), Die Kopfregion der Kranioten beim Amphioxus, nebst Bemerkungen über die Wirbeltheorie des Schädels, in: Anat. Anz., Vol. 4. 